\section{Changes in lamina cribrosa and prelaminar tissue after deep sclerectomy}

C Barrancos, G Rebolleda, N Oblanca, C Cabarga and FJ Muñoz-Negrete
Hospital Ramón y Cajal, Department of Ophthalmology, Madrid, Spain

Correspondence: C Barrancos, Hospital Ramón y Cajal, Carretera de Colmenar Viejo $\mathrm{km}$ 9,100, CP 28034, Madrid, Spain.

Tel: + 3491 3368886; Fax: + 34913368126 E-mail: constanzabj@ yahoo.es

This work has been previously presented at the Spanish Glaucoma Society in Córdoba (Spain), 1 March 2013.

Received: 1 May 2013 Accepted in revised form: 27 September 2013 Published online: 15 November 2013

\begin{abstract}
Aim To determine the response of the lamina cribrosa (LC) and prelaminar tissue to a reduction of intraocular pressure (IOP) after nonpenetrating deep sclerectomy (NPDS) using enhanced depth imaging (EDI) spectral domain optical coherence tomography (SD-OCT).

Methods A total of 28 eyes from 28 patients presenting with primary open angle glaucoma who underwent NPDS were studied. SD-OCT scans using EDI technology were obtained before surgery and 1 week, 1 month, and 3 months postoperatively. The OCT device was set to image a $15 \times 10^{\circ}$ vertical rectangle centred on the optic disc. The scan closest to the optic nerve head $(\mathrm{ONH})$ centre was selected for analysis. The vertical distances from three equidistant points on the reference line (Bruch's membrane opening) to the anterior prelaminar tissue surface and the anterior and posterior surfaces of the LC were measured.

Results The IOP decreased from $18.7 \pm 4.3$ to $9.1 \pm 4.0$ at the first week, $11.4 \pm 3.7$ at 1 month, and $13.1 \pm 3.6 \mathrm{~mm} \mathrm{Hg}$ at 3 months postoperatively $(P<0.001)$. There was a significant reduction of the $\mathrm{ONH}$ cupping at 1 week (22.3\%, P<0.001), 1 month (13.7\%, $P<0.001)$, and 3 months $(9.8 \%, P=0.001)$ after surgery. Anterior displacement of the LC was slight but statistically significant at 1 week (4.5\%, $P=0.003)$, 1 month (3.8\%, $P=0.014)$, and 3 months postoperatively $(3.3 \%$, $P=0.010$ ). IOP reduction was significantly correlated with a reduction of $\mathrm{ONH}$ cupping and anterior displacement of LC at the first week and first month $(P<0.05)$.

Conclusions Cupping reversal after NPDS is mainly due to changes in prelaminar tissue thickness, whereas the LC changes in position are less pronounced.
\end{abstract}

Eye (2014) 28, 58-65; doi:10.1038/eye.2013.238; published online 15 November 2013

Keywords: lamina cribrosa; prelaminar tissue; optic nerve head; glaucoma; deep sclerectomy

Introduction

The lamina cribrosa (LC) is thought to have an essential role in the physiopathology of glaucoma. ${ }^{1,2}$ It is composed of a delicate net of connective tissue through which the axons of the retina ganglion cells exit the eye and provides both structural and functional support for the axons while constituting a barrier between the high-pressure intraocular environment and the low-pressure retrobulbar space. $^{3}$

The evolution in imaging devices during the last few decades has allowed for a better understanding of the optic nerve head $(\mathrm{ONH})$ structure. Optical coherence tomography (OCT) is a high-resolution imaging technique that applies the principle of interferometry to acquire in vivo measurements of retinal nerve fibre layer (RNFL) thickness. It has progressed from the initial temporal domain devices to spectral domain (SD) OCT, offering increased speed and resolution for imaging. Enhanced depth imaging (EDI) technology has led to a revolution in the study of deeper eye structures, such as the choroid and the LC. ${ }^{4-6}$ It allows visualisation of the LC underneath the neuroretinal ring and provides high resolution images similar to those obtained by histology. This technique has been used to evaluate the LC in both normal and glaucomatous subjects. ${ }^{7,8}$

The LC is a dynamic structure that is modified by intraocular pressure (IOP) changes. Optic disc cupping reversal after IOP reduction has been documented in several studies by 
photography and confocal scanning laser tomography. ${ }^{9-12}$ In the last few years, the advent of the newest OCT devices has improved evaluation of these changes, including the visualisation of the posterior face of the LC. Recently, some studies have reported changes in $\mathrm{ONH}$ morphology after trabeculectomy and tube shunt implantation that were assessed by the OCT with EDI technique. ${ }^{13-15}$ However, to the best of our knowledge, the changes in $\mathrm{ONH}$ morphology after nonpenetrating deep sclerectomy (NPDS) glaucoma surgery have not yet been reported.

The current study evaluates changes in the thickness and relative position of laminar and prelaminar tissues in the ONH after IOP lowering by NPDS glaucoma surgery as observed by EDI SD-OCT. Contrary to trabeculectomy or tube shunt implantation, NPDS avoids a sudden ocular decompression. A gentler rate of IOP drop may influence the response of the $\mathrm{ONH}$ to pressure changes. The main purpose of this study is to assess the changes in ONH cupping after NPDS and evaluate whether this change depends predominantly on laminar or prelaminar modifications.

\section{Materials and methods}

\section{Participants}

Patients with primary open angle glaucoma (POAG) who had been clinically selected for NPSD between January and October 2012 were selected for enrolment in this prospective, observational study. A total of 41 out of the 50 patients initially selected agreed to participate.

The study was approved by Hospital Ramón y Cajal Review Board. All participants provided written informed consent according to the Declaration of Helsinki.

To be included, patients were required to have a best corrected visual acuity better than 20/70. POAG was defined by the presence of glaucomatous optic disc damage (localised or diffuse neuroretinal rim thinning or RNFL defect) associated with typical, reproducible visual field defects, defined as glaucoma hemifield test results outside normal limits on at least two consecutive visual field tests within a 6-month period or the presence of at least three contiguous test points within the same hemifield on the pattern deviation plot at $P<0.01$, with at least one point at $P<0.005$. Visual field test reliability indices better than $25 \%$ were required to be included in the study.

Glaucomatous eyes comorbid with optic disc drusen, anterior ischaemic optic neuropathy, retinal diseases such as retinal vessel occlusion or diabetic retinopathy, or neurologic diseases such as pituitary tumour were excluded. Eyes were also excluded if they had a history of ocular surgery other than cataract extraction or if a good-quality image (ie, quality score 15) could not be obtained. Patients unable to cooperate with the imaging protocol due to ocular fixation issues were excluded. Patients presenting an IOP $\leq 3 \mathrm{~mm} \mathrm{Hg}$ at the first week postoperative follow-up were excluded to avoid measurement artefacts.

\section{Image acquisition protocol}

The optic disc was evaluated using Spectralis OCT (Heidelberg Engineering GmbH, Heidelberg, Germany) and the EDI technique. The OCT device was set to image a $15 \times 10$ degree vertical rectangle centred on the optic disc. Thirteen cross-section scans were obtained, which were $\sim 120 \mu \mathrm{m}$ apart. Each section had 70 OCT frames averaged. Images were obtained through undilated pupils during the preoperative period (between 10 and 1 days preoperatively) and 1 week, 1 month, and 3 months postoperatively. Images of the postoperative period were obtained with the 'Follow-up' protocol provided by Spectralis OCT, allowing the evaluation of changes at the same location.

\section{Measurement of the thickness and relative position of the LC and prelaminar tissue}

One vertical scan that was the closest to the ONH centre and did not include any main vessels (retina central artery or vein) was selected from the preoperative images. The same scan was analysed in subsequent exams. A reference line connecting Bruch's membrane opening (BMO) was drawn using the calipers provided by the Spectralis OCT software. Three equidistant points, corresponding to one-half and one-thirds of the reference, were highlighted at the reference line. They were selected because of their reproducibility and because they would avoid measurement on the neuroretinal rim. Vertical lines connecting these three points on the reference line to the anterior face of the prelaminar tissue and the anterior and posterior surfaces of the LC were obtained and measured. ONH excavation was defined as the average of the length of the three lines connecting the reference line to the anterior face of the prelaminar tissue. Relative LC position was defined as the average of the length of the three lines connecting the reference line to the anterior face of the LC. Prelaminar tissue thickness was defined as the difference between the position of the anterior face of the prelaminar tissue and the anterior face of the LC. LC thickness was defined as the difference between the position of the anterior and posterior faces of the LC.

All of the measurements were carried out by the same observer (C.B.J.). The observer was blind to the clinical parameters and the time period (preoperative or follow-up) 
for each scan when assessing them. To evaluate intraobserver reproducibility, 16 randomly selected scans were remeasured by the same observer, and the intraclass correlation was calculated.

\section{Statistical analyses}

Statistical analyses were performed using SPSS software (version 16.0; SPP Inc., Chicago, IL, USA). The calculated power of the study was $80 \%$ when designed. Given the small sample size, the nonparametric test was used.

$P<0.05$ was considered significant.

The preoperative and postoperative amount of prelaminar and laminar relative position and thickness were compared using the non-parametric Wilcoxon test. Spearman correlation analyses were performed to adjust the changes found in the ONH to other factors such as IOP, RNFL thickness, mean perimetry deviation, age or axial length (AL).

\section{Results}

We enrolled 28 eyes from 28 patients (53.6\% women) undergoing NPDS with a mean age of $66.5 \pm 11.9$ years. The mean central corneal thickness was $538.8 \pm 28.2 \mu \mathrm{m}$, the preoperative mean perimetry deviation was $-14.8 \pm 8.7 \mathrm{~dB}$, the mean RNFL thickness was $60.6 \pm 16.6 \mu \mathrm{m}$, and the mean AL was $24.3 \pm 2.5 \mathrm{~mm}$ (Table 1).

From the 41 patients initially enrolled, 13 had to be excluded. Seven patients were excluded owing to poor image quality. Four patients were excluded owing to intraoperative rupture of the trabeculo-Descemet's membrane. Two patients were excluded owing to postoperative severe hypotony (IOP $\leq 3 \mathrm{~mm} \mathrm{Hg}$ at the first week postoperative follow-up).

There was a statistically significant decrease in the mean IOP from $18.7 \pm 4.3 \mathrm{~mm} \mathrm{Hg}$ to $9.1 \pm 4.0$ at the first week, $11.4 \pm 3.7$ at 1 month, and $13.1 \pm 3.6 \mathrm{~mm} \mathrm{Hg}$ at 3 months postoperatively $(P<0.001)$ (Table 2$)$.

The intraclass correlation coefficients for measurement of the ONH excavation and position of the anterior and posterior surfaces of the LC were $0.999,0.978$, and 0.564 , respectively. Although the posterior surface of the LC was seen in most of the images, determination of its precise limits was difficult in many instances. Owing to a low correlation coefficient at the measurement of the posterior surface of the LC, this parameter was not analysed any further in this study.

We observed a significant reduction of the $\mathrm{ONH}$ excavation at 1 week $(22.3 \%, P<0.001), 1$ month $(13.7 \%$, $P<0.001)$, and 3 months after surgery $(9.8 \%, P=0.001)$. Anterior displacement of the LC was limited but statistically significant at 1 week $(4.5 \%, P=0.003)$,
1 month $(3.8 \%, P=0.014)$, and 3 months postoperatively (3.3\%, $P=0.010)$. Prelaminar tissue thickness significantly increased at the first week $(23.6 \%, P<0.001)$, the first month $(9.9 \%, P<0.003)$, and the third month postoperatively $(6.4 \%, P<0.016)$. (Table 2, Figure 1).

The mean diameter of the BMO was 1649.6 (329.8) $\mu \mathrm{m}$. No significant changes during the follow-up at the first week $(P=0.475)$, first month $(P=0.648)$, and third month $(P=0.966)$ were observed. We did observe a slight increase $(1.82 \mu \mathrm{m})$ in RNFL thickness within the first week $(P=0.013)$. This change was reversed in the first $(P=0.292)$ and third months $(P=0.793)$ (Table 2$)$.

Using Spearman correlation analysis, we found a positive correlation between IOP reduction and the reduction of $\mathrm{ONH}$ excavation at 1 week $(0.398, P=0.036)$ and at 1 month $(0.502, P=0.006)$. LC anterior displacement also showed a correlation with IOP reduction at 1 week $(0.435, P=0.021)$ and 1 month $(0.417$, $P=0.027)$. These changes were not statistically significant at the third month $(P=0.408, P=0.483)$. IOP reduction and increased prelaminar tissue thickness were not statistically correlated ( 1 week: $0.190, P=0.333 ; 1$ month: $0.239, P=0.221 ; 3$ months: $0.159, P=0.449)$.

No significant correlation was found between the structural changes in ONH and age, AL, RNFL thickness, or pachymetry at any follow-up time (Table 3).

\section{Discussion}

Optic nerve cupping reversal after acute reduction of IOP has long been described in patients undergoing surgery or after medical treatment. ${ }^{10-12}$ These changes might be due to an increase in the prelaminar tissue thickness, an anterior displacement of the LC or a combination of both features. Different authors have supported different hypotheses regarding this controversy.

Parrish II et $a l^{10}$ studied the effect of IOP lowering in the $\mathrm{ONH}$, assessing baseline and 5 -year follow-up stereoscopic disc photographs in POAG patients. Eyes that received surgical treatment and those with lower postoperative IOP were more likely to develop cupping reversal. They suggested that the reversal likely followed an anterior movement of the LC and papillary changes with the possibility of a thickening of the prelaminar neural tissue.

In a series of 35 glaucoma patients undergoing trabeculectomy, Lee et $a^{13}$ found that the reversal of optic nerve cupping was primarily a result from the anterior movement of the LC, although the changes in prelaminar tissue and LC thickness were less remarkable. Reis et al ${ }^{15}$ suggested that the prelaminar tissue thickening and anterior displacement of the LC are both responsible for the changes in the $\mathrm{ONH}$ after IOP reduction secondary to trabeculectomy or tube shunt implantation. 
Table 1 Patient demographics and clinical characteristics $(n=28)$

\begin{tabular}{lrrc}
\hline & Mean & \multicolumn{1}{c}{ SD } & Range \\
\hline Age (years) & 66.5 & 11.9 & $30-84$ \\
CCT $(\mu \mathrm{m})$ & 538.8 & 28.2 & $485-590$ \\
Perimetry deviation $(\mathrm{dB})$ & -14.8 & 8.7 & -0.81 to -30.82 \\
RNFL $(\mu \mathrm{m})$ & 60.6 & 16.6 & $35-89$ \\
AL $(\mathrm{mm})$ & 24.3 & 2.5 & $21.8-34.6$ \\
\hline
\end{tabular}

Those changes were more remarkable at the 1- and 3 -month follow-ups, in accord with the greater IOP reduction observed at these time points.

Our results show that the morphological changes in ONH after NPDS represent mostly decompression of the prelaminar tissue, as well as a mild anterior laminar displacement. Agoumi et $a l^{16}$ have previously reported the opposite $\mathrm{ONH}$ changes after acute IOP elevation (compression of prelaminar tissue with limited posterior displacement of LC), which supports the same hypothesis.

The correlations between the changes in the position and thickness of the LC and the prelaminar tissue and IOP variation have already been studied, showing variable results. There are some differences among the studies that might explain the different results. Lee et $a l^{13}$ reported that cupping reversal was mainly a result from the anterior movement of the LC. However, Reis et a $l^{15}$ found that prelaminar tissue thickening and anterior displacement of the LC are both responsible. In the current study, both the mean preoperative IOP (18.7 vs $27.2 \mathrm{~mm} \mathrm{Hg}$ ) and the mean postoperative reduction $(9.6$ vs $16.7 \mathrm{~mm} \mathrm{Hg}$ ) were lower compared with Lee's study. On the contrary, IOP measurements were comparable to Reis' study (mean preoperative IOP $18.1 \mathrm{~mm} \mathrm{Hg}$; mean postoperative reduction $6.7 \mathrm{~mm} \mathrm{Hg}$ ). Moreover, our patients underwent NPDS surgery, which generally produces a lower IOP reduction compared with trabeculectomy or tube shunt implantation. ${ }^{17-19}$ This suggests that the LC displacement is associated with larger IOP reductions, whereas milder changes primarily affect the prelaminar tissue. However, Lee $e t$ al also included 12 patients who had preoperative IOPs within the normal range $(18.2 \mathrm{~mm} \mathrm{Hg})$, and they found significant cupping reversal and anterior displacement of the LC. In contrast to our study, they do not describe remarkable changes in the prelaminar tissue thickness. This variability in the $\mathrm{ONH}$ response to IOP reduction could be due to small sample sizes, the surgical technique or to the presence of coexisting modifying factors.

One of the advantages of NPDS is that it avoids sudden decompression of the IOP, which is a more characteristic feature of other glaucoma surgeries like

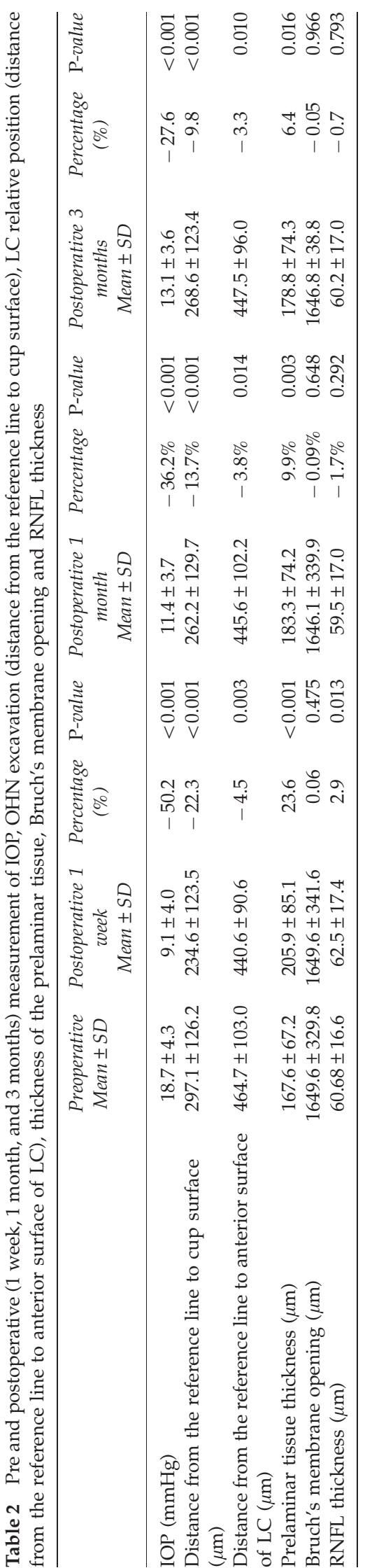



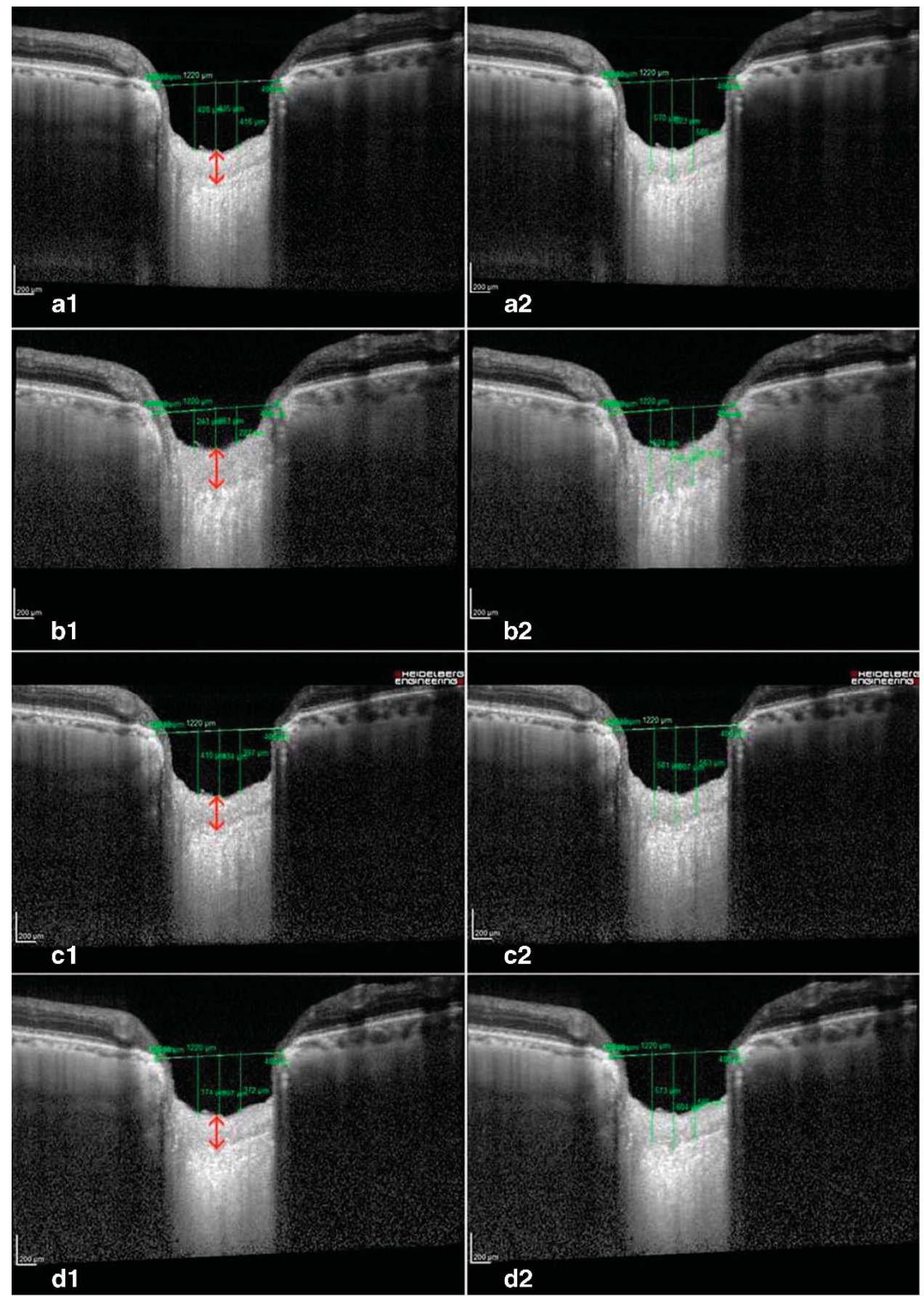

Figure 1 Images obtained preoperatively (a1, a2), and at 1 week (b1, b2), 1 month (c1, c2), and 3 months (d1, d2) postoperatively. The IOP decreased from 18 to $6 \mathrm{~mm} \mathrm{Hg}$ at 1 week, $13 \mathrm{~mm} \mathrm{Hg}$ at 1 month, and $14 \mathrm{~mm} \mathrm{Hg}$ at 3 months after surgery. a reference line connecting the termination of bruch's membrane was set (horizontal lines). Three vertical lines connecting points from the reference line to cup surface (a1, b1, c1, d1) and to the anterior surface of the Lc (a2, b2, c2, d2) were measured (vertical lines). Thickening of the prelaminar tissue (red arrow) and cupping reversal are noticeable in b1, but laminar displacement is mild (b2). Prelaminar thickness and cup excavation decreased at 1 month (c1, c2) and 3 months postoperatively (d1, d2).

trabeculectomy or tube shut implantation. IOP drop occurs more gradually in NPDS compared with the other two techniques, but this does not necessarily jeopardise the surgery effectiveness. NPDS has probed to provide an adequate IOP lowering. ${ }^{20,21}$ Our patients suffered an IOP drop from an average of 18.7 to $9.6 \mathrm{~mm} \mathrm{Hg}$ in the 
Table 3 Spearman correlation analysis on IOP reduction and reduction of the distance from the reference line to cup surface, reduction of the distance from the reference line to the anterior surface of the LC, and increase of prelaminar tissue thickness at first week, 1 , and 3 months

\begin{tabular}{|c|c|c|c|}
\hline & \multicolumn{3}{|c|}{ IOP reduction } \\
\hline & 1 week & 1 month & 3 months \\
\hline \multirow[t]{2}{*}{ Reduction of the distance from the reference line to cup surface } & 0.398 & 0.502 & 0.173 \\
\hline & $P=0.036$ & $P=0.006$ & $P=0.408$ \\
\hline \multirow[t]{2}{*}{ Reduction of the distance from the reference line to anterior surface of LC } & 0.435 & 0.417 & 0.147 \\
\hline & $P=0.021$ & $P=0.027$ & $P=0.483$ \\
\hline \multirow[t]{2}{*}{ Increase in prelaminar tissue thickness } & 0.190 & 0.239 & 0.159 \\
\hline & $P=0.333$ & $P=0.221$ & $P=0.449$ \\
\hline
\end{tabular}

Bold numbers show statistical significance.

first postoperative week. This is a relatively decent decompression, but is not an uncommon result in NPDS surgeries. A pressure of around $5 \mathrm{~mm} \mathrm{Hg}$ in the early postoperative days is generally a favourable predictive factor for a successful operation, indicating that the dissection was carefully and efficiently performed. However, this initial hypotony should not be prolonged for $>1$ or 2 weeks. ${ }^{20}$ In our study, patients with postoperative IOP $\leq 3 \mathrm{~mm} \mathrm{Hg}$ at the first week or Descemet membrane rupture were excluded from the analysis of the data. We agree that one of the main advantages of NPDS is the avoidance of hypotony, but even if uncommon, it is a possible postoperative complication. To prevent any loss in the data validity, those two cases where excluded.

We believe that the different surgical technique might be one factor explaining the different results in the postoperative ONH changes between our work and Lee's and Reis' studies. Trabeculectomy and tube shunt implantation, producing a rapid ocular decompression, could induce greater changes in the LC position. NPDS, producing a gradual IOP drop, might generate a milder anterior displacement of the LC. Consequently, even if there might be certain correlations between IOP reduction and structural changes, LC displacement could be dependent on multifactorial variables such as the magnitude of the IOP changes, the surgery technique or the baseline ONH configuration.

Our study suggests that early cupping reversal is mainly due to a postoperative increase in prelaminar tissue. However, unlike the reduction of ONH excavation and LC anterior displacement, the increase in prelaminar tissue did not show a statistical correlation with IOP reduction. Once again, we believe that multifactorial causes account for the variable response of prelaminar tissues to IOP reduction. The main factor could be the presence of postoperative oedema; however, we do not believe that the presence of papillary oedema was the principal cause of postoperative prelaminar tissue thickening given that the average RNFL thickness only changed by $1.82 \mu \mathrm{m}$ within the first week (preoperative mean RNFL: $60.68 \pm 16.6 \mu \mathrm{m}, 1$ week postoperative mean RNFL: $62.5 \pm 17.4 \mu \mathrm{m}, P=0.013)$. Individual responses to ocular inflammation and surgery can modify the presence of oedema. Other influencing factors in the response of prelaminar tissue to IOP reduction could be the preoperative prelaminar tissue thickness, preoperative RNFL thickness or degree of glaucoma damage.

We found a positive correlation between the mean deviation at perimetry and the reduction of excavation (Pearson coefficient $0.963, P=0.037$ ) and between the mean deviation and increased prelaminar tissue thickness (Pearson coefficient 0.969, $P=0.031$ ), both within the first week. Therefore, patients showing less glaucoma damage presented a higher reduction in $\mathrm{ONH}$ excavation. Structural changes of the LC and its surrounding tissues during the development and progression of glaucoma have already been described. ${ }^{22,23}$ On the other hand, we did not find significant statistical correlation between structural changes and preoperative prelaminar thickness or RNFL thickness.

Age could be another modifying factor in the response of the ONH. The mean age in our study (66.5 years) was comparable to that in Reis' study (71.4 years) but was remarkably older than that for Lee's sample (52.6 years). Several studies have suggested that the LC is stiffer in older ONHs. ${ }^{24,25}$ This lower elasticity might be the cause of the reduced anterior LC displacement that we found, compared to Lee's study. However, we did not find a correlation between age and structural changes in our series.

Our study included seven myopic eyes (25.0\%) having an AL over $25 \mathrm{~mm}$ (27.24 $\pm 3.37 \mathrm{~mm}$, range 25.02$34.69 \mathrm{~mm}$ ). LC thickness has been reported to not correlate with AL in healthy eyes, ${ }^{26}$ but the LC could have different elasticity properties in myopic eyes and therefore respond differently to IOP changes. In highly myopic eyes, the LC is significantly thinner than in nonhighly myopic eyes, which decreases the distance 
between the intraocular space and the cerebrospinal fluid space and steepens the translaminar pressure gradient at a given IOP. This has been presented as an explanation for the increased susceptibility to glaucoma in highly myopic eyes. ${ }^{27}$ In the current study, no correlation was found between AL and structural changes.

This study has some limitations. First, our follow-up finished at the third month, as we did not expect to find significant changes over the medium term because the reversal of the changes observed at the first week occurred mainly within the first month, and remarkable changes were not observed at the third month.

Second, even if pre- and postoperative scans were acquired at the same location using the 'follow-up' acquisition protocol, the reference line was set manually, which may affect the obtained measurements. There might be slight changes in the position or angle of the reference line, thus introducing a measurement error. However, we did not find an angle misalignment $>10^{\circ}$, most likely because the measurements were masked, thus reducing the possibility of biases.

Third, the laminar structures were assessed on one vertical scan selected at the centre of the ONH, while the remaining peripheral scans were not evaluated. Even though a global evaluation of the ONH would have been desirable, we believe that this scan is representative due to the consistency of the measurement technique used in this study; the same segment of the $\mathrm{ONH}$ was evaluated both pre- and postoperatively, allowing us to assess the structural changes at overlapping points.

Fourth, only 28 patients completed the study. A larger sample would be desirable to strengthen the results of the study, but we believe that our data provides an initial approach to understanding the effects of NPDS on the ONH. To our knowledge, the changes in ocular parameters after deep sclerectomy have not been previously reported. Without a control population, we cannot completely dismiss any temporal fluctuation in non-surgical patients, but we have not clinically observed these changes in our practice.

Our findings suggest that early cupping reversal after NPDS is mainly due to changes in prelaminar tissue thickness, whereas the LC changes in position are less pronounced. We suggest that the prelaminar tissue might act similar to a buffer for pressure changes. It compresses when the IOP increases and becomes thicker when IOP decreases. Further studies are needed to determine the influence of several factors on the long-term response of the ONH structures to IOP changes.

\section{Summary}

What was known before

- Lamina cribrosa changes after trabeculectomy and tube shunt implantation have already been described.

- They found that reversal of the optic disc cupping is mainly due to an anterior displacement of the lamina cribrosa.

What this study adds

- We study the changes in the lamina cribrosa and prelaminar tissue after deep sclerectomy.

- We suggest that cupping reversal after deep sclerectomy is mainly due to changes in prelaminar tissue thickness, whereas the LC changes in position are less pronounced.

\section{Conflict of interest}

The authors declare no conflict of interest.

\section{Acknowledgements}

The first author (Constanza Barrancos) received a grant from Jesús de Gangoiti Barrera Foundation to perform this study. The Foundation has no proprietary or commercial interest in any of the material discussed in this article.

\section{References}

1 Burgoyne CF, Downs JC, Bellezza AJ, Suh JK, Hart RT. The optic nerve head as a biomechanical structure: a new paradigm for understanding the role of IOP-related stress and strain in the pathophysiology of glaucomatous optic nerve head damage. Prog Retin Eye Res 2005; 24: 39-73.

2 Quigley HA, Addicks EM, Green WR, Maumenee AE. Optic nerve damage in human glaucoma. II. The site of injury and susceptibility to damage. Arch Ophthalmol 1981; 99: 635-649.

3 Morgan WH, Yu DY, Cooper RL, Alder VA, Cringle SJ, Constable IJ. The influence of cerebrospinal fluid pressure on the lamina cribrosa tissue pressure gradient. Invest Ophthalmol Vis Sci 1995; 36: 1163-1172.

4 Yeoh J, Rahman W, Chen F, Hooper C, Patel P, Tufail A et al. Choroidal imaging in inherited retinal disease using the technique of enhanced depth imaging optical coherence tomography. Graefes Arch Clin Exp Ophthalmol 2010; 248: 1719-1728.

5 Dell'Omo R, Costagliola C, Di Salvatore F, Cifariello F, Dell'Omo E. Enhanced depth imaging spectral-domain optical coherence tomography. Retina 2010; 30: 378-379.

6 Lee EJ, Kim TW, Weinreb RN, Park KH, Kim SH, Kim DM. Visualization of the lamina cribrosa using enhanced depth imaging spectral-domain optical coherence tomography. Am J Ophthalmol 2011; 152: 87-95; e81.

7 Park SC, De Moraes CG, Teng CC, Tello C, Liebmann JM, Ritch R. Enhanced depth imaging optical coherence tomography of deep optic nerve complex structures in glaucoma. Ophthalmology 2012; 119: 3-9. 
8 Park HY, Jeon SH, Park CK. Enhanced depth imaging detects lamina cribrosa thickness differences in normal tension glaucoma and primary open-angle glaucoma. Ophthalmology 2012; 119: 10-20.

9 Yuen D, Buys YM. Disc photography and Heidelberg retinal tomography documentation of reversal of cupping following trabeculectomy. Graefes Arch Clin Exp Ophthalmol 2010; 248: 1671-1673.

10 Parrish 2nd RK, Feuer WJ, Schiffman JC, Lichter PR, Musch DC. Five-year follow-up optic disc findings of the Collaborative Initial Glaucoma Treatment Study. Am J Ophthalmol 2009; 147: 717-724; e711.

11 Irak I, Zangwill L, Garden V, Shakiba S, Weinreb RN. Change in optic disk topography after trabeculectomy. Am J Ophthalmol 1996; 122: 690-695.

12 Park KH, Kim DM, Youn DH. Short-term change of optic nerve head topography after trabeculectomy in adult glaucoma patients as measured by Heidelberg retina tomograph. Korean J Ophthalmol 1997; 11: 1-6.

13 Lee EJ, Kim TW, Weinreb RN. Reversal of lamina cribrosa displacement and thickness after trabeculectomy in glaucoma. Ophthalmology 2012; 119: 1359-1366.

14 Lee EJ, Kim TW, Weinreb RN, Kim H. Reversal of lamina cribrosa displacement after intraocular pressure reduction in open-angle glaucoma. Ophthalmology 2013; 120: 553-559.

15 Reis AS, O'Leary N, Stanfield MJ, Shuba LM, Nicolela MT, Chauhan BC. Laminar displacement and prelaminar tissue thickness change after glaucoma surgery imaged with optical coherence tomography. Invest Ophthalmol Vis Sci 2012; 53: 5819-5826.

16 Agoumi Y, Sharpe GP, Hutchison DM, Nicolela MT, Artes PH, Chauhan BC. Laminar and prelaminar tissue displacement during intraocular pressure elevation in glaucoma patients and healthy controls. Ophthalmology 2011; 118: 52-59.

17 Chiselita D. Non-penetrating deep sclerectomy versus trabeculectomy in primary open-angle glaucoma surgery. Eye (Lond) 2001; 15: 197-201.
18 Mermoud A, Schnyder CC, Sickenberg M, Chiou AG, Hédiguer SE, Faggioni R. Comparison of deep sclerectomy with collagen implant and trabeculectomy in open-angle glaucoma. J Cataract Refract Surg 1999; 25: 323-331.

19 Gedde SJ, Schiffman JC, Feuer WJ, Herndon LW, Brandt JD, Budenz DL et al. Three-year follow-up of the tube versus trabeculectomy study. Am J Ophthalmol 2009; 148: 670-684

20 Shaarawy T, Flammer J, Smits G, Mermoud A. Low first postoperative day intraocular pressure as a positive prognostic indicator in deep sclerectomy. Br J Ophthalmol 2004; 88: 658-661.

21 Karlen ME, Sanchez E, Schnyder CC, Sickenberg M, Mermoud A. Deep sclerectomy with collagen implant: medium term results. Br J Ophthalmol 1999; 83: 6-11.

22 Grytz R, Sigal IA, Ruberti JW, Meschke G, Downs JC. Lamina cribrosa thickening in early glaucoma predicted by a microstructure motivated growth and remodeling approach. Mech Mater 2012; 44: 99-109.

23 Grytz R, Girkin CA, Libertiaux V, Downs JC. Perspectives on biomechanical growth and remodeling mechanisms in glaucoma(). Mech Res Commun 2012; 42: 92-106.

24 Leung LK, Ko MW, Lam DC. Effect of age-stiffening tissues and intraocular pressure on optic nerve damages. Mol Cell Biomech 2012; 9: 157-173.

25 Albon J, Purslow PP, Karwatowski WS, Easty DL. Age related compliance of the lamina cribrosa in human eyes. Br J Ophthalmol 2000; 84: 318-323.

26 Lee EJ, Kim TW, Weinreb RN, Suh MH, Kim H. Lamina cribrosa thickness is not correlated with central corneal thickness or axial length in healthy eyes: central corneal thickness, axial length, and lamina cribrosa thickness. Graefes Arch Clin Exp Ophthalmol 2013; 251: 847-854.

27 Jonas JB, Berenshtein E, Holbach L. Lamina cribrosa thickness and spatial relationships between intraocular space and cerebrospinal fluid space in highly myopic eyes. Invest Ophthalmol Vis Sci 2004; 45: 2660-2665. 